\title{
Involvement of Erythrocyte Calpain in Glycine- and Carnitine-Treated Isovaleric Acidemia
}

\author{
FRANCA SALAMINO, FABIO DI LISA, ALBERTO B. BURLINA, ROBERTA MENABŎ, \\ ROBERTA BARBATO, ROBERTO DE TULLIO, AND NORIS SILIPRANDI \\ Istituto di Chimica Biologica, Università di Genova, 16132 Genova, Italy [F.S., R.D.T.]; \\ Dipartimento di Chimica Biologica, Università di Padova and Centro Studio Fisiopatologia \\ Mitocondriale, CNR, 35121 Padova, Italy [F.D.L., R.M., R.B., N.S.]; and and Dipartimento di \\ Pediatria, Università di Padova, 35128 Padova, Italy [A.B.B]
}

\section{ABSTRACT}

\begin{abstract}
When a 12-y-old girl suffering from isovaleric acidemia was treated with L-carnitine, there was a considerable increase in her blood and urine concentration of isovalerylcarnitine. When later the patient received an infusion of glycine in place of carnitine, isovalerylcarnitine reverted toward the low levels found in a normal subject. At the end of either treatment, erythrocyte calpain was measured and found to be decreased after carnitine therapy (140 versus 96 $\mathrm{U} / \mathrm{mg} \mathrm{Hb}$ with glycine or carnitine, respectively). Because we have previously shown that the activity of calpain isolated from erythrocytes was markedly modified by isovalerylcarnitine, the present results might be seen as the
\end{abstract}

The intracellular nonlysosomal calcium-activated cysteine proteinases, commonly referred as calpains or calcium-activated proteinases, are present in virtually every eukaryotic cell type. On the basis of their sensitivity for $\mathrm{Ca}^{2+}$, calpains have been subdivided into $\mu$-calpain and m-calpain, requiring 5-50 $\mu \mathrm{M} \mathrm{Ca}^{2+}$ and $0.2-0.6 \mathrm{mM}$ $\mathrm{Ca}^{2+}$, respectively, for half maximum activity (1-5).

We have recently found that IVC, a product of leucine catabolism, is a potent activator of the m-calpains, but not the $\mu$-calpains, isolated from various rat tissues $(6,7)$. This activation comprises a 10 -fold increase in the $\mathrm{Km}$ of calpain for $\mathrm{Ca}^{2+}$ together with an increase in the $\mathrm{V}_{\max }$ above the values observed with the native enzyme at saturating $\mathrm{Ca}^{2+}$ concentrations. It is additional to the activation produced by adding phospholipid vesicles, showing that IVC could be acting as a highly selective activator of calpain, whether cytosolic or membrane bound. These effects were highly specific for the L-isomer of IVC. The D-isomer, other branched-chain acylcar-

\footnotetext{
Received September 21, 1993; accepted March 3, 1994.

Correspondence and reprint requests: Prof. Noris Siliprandi, Dipartimento di Chimica Biologica, Via Trieste, 75, 35121 Padova, Italy.

Supported by CNR target project "Prevention and Control of Disease Factors" (SP8, Control of cardiovascular diseases) (Grant 91000237; PF 41), Telethon, Italy (Grant 378), and Sigma Tau (Pomezia, Italy).
}

consequence of the chronic exposure of the patient's red blood cells to high levels of isovalerylcarnitine. The lowered calpain activity was also proved by an increase in erythrocyte band 3 phosphorylation together with an increased erythrocyte fragility after calcium loading in the presence of the ionophore A-23187. Calpastatin, the natural inhibitor of calpain, was only slightly modified. (Pediatr Res 36: 182-186, 1994)

IVC, isovalerylcarnitine

Abbreviations

nitines, and palmitoylcarnitine (6) as well as acetylcarnitine and propionylcarnitine, short-chain acyl-CoA, and fatty acids (Pontremoli S, Melloni E, Michetti M, Sparatore B, Salamino F, Siliprandi N, Horecker BL, unpublished experiments) were not effective.

It should be outlined again that these results have been obtained in vitro on calpains purified from tissues or cells. Attempts to confirm whether this action could be seen in a more organized system have failed. For instance, no action on calpain has been observed by incubating human neutrophils in the presence of $2 \mathrm{mM}$ IVC for $15 \mathrm{~min}$ at $30^{\circ} \mathrm{C}$. One reason for this failure could be the impossibility of obtaining in vitro the sufficient length of exposure, a condition that might be conveniently achieved in vivo.

Because under physiologic conditions IVC is almost undetectable in both plasma and urine, the problem of a possible activation of calpain in vivo by this carnitine ester does not exist.

A condition implying a significant accumulation of IVC is isovaleric acidemia, especially when the patient is treated with a large amount of carnitine $(8,9)$. In this condition, blood and tissue cells are chronically exposed to abnormally high concentrations of IVC, as is the case for the patient described in the present paper. 
In a girl affected by isovaleric acidemia, the activity of erythrocyte calpain has been comparatively evaluated either after a 6-mo period of carnitine treatment or after the same period of glycine treatment. We found that carnitine treatment actually decreases erythrocyte calpain activity, providing evidence that the decrease in calpain activity is in fact caused by its initial activation by IVC. Once activated, calpain undergoes autodigestion with a progressive decrease in its activity (10). The observed decrease could therefore be caused by the initial activation of erythrocyte calpain activity by the increased IVC (6).

\section{METHODS}

Clinical description and study. C.S., now a 12-y-old female, came to our attention 3 y ago after a short history of persisting vomiting. She presented with muscular atrophy and lethargy. Routine laboratory tests revealed hyperammonemia $(83 \mu \mathrm{mol} / \mathrm{L})$, hyperuricemia $(0.62$ $\mu \mathrm{mol} / \mathrm{L}$ ), and metabolic acidosis. The diagnosis of isovaleric acidemia was based on the urinary excretion of isovalerylglycine and 3-hydroxyisovaleric acid. Her blood carnitine concentration was extremely low $(<5$ $\mu \mathrm{mol} / \mathrm{L})$.

Despite oral L-carnitine therapy $(100 \mathrm{mg} / \mathrm{kg} / \mathrm{d})$ and a controlled protein intake $(40 \mathrm{~g} / \mathrm{d})$, her plasma total carnitine concentration remained low $(15 \mu \mathrm{mol} / \mathrm{L})$ and was normalized after increasing the dosage to $200 \mathrm{mg} / \mathrm{kg} / \mathrm{d}$. No additional episodes of vomiting and lethargy were observed.

Blood and urine samples were first collected, then the carnitine was stopped and immediately replaced with glycine $(250 \mathrm{mg} / \mathrm{kg} / \mathrm{d})$. After 6 mo of glycine treatment, blood and urine samples were collected again. No major clinical differences were observed as a result of exchanging carnitine for glycine. Control blood and urine samples were obtained from untreated healthy subjects of the same sex and age.

Metabolite assays. Free carnitine was determined by a radioenzymatic method (11) in plasma (after perchloric acid deproteinization) and in urine (after chloroform extraction). Total carnitine was assayed after alkaline hydrolysis. The acid-soluble fraction was also assayed radioenzymatically for acetylcarnitine (12) and for shortchain acyl esters by HPLC (13). Briefly, radioactive carnitine was exchanged to isotopic equilibrium into the acylcarnitine pool of the sample $(0.5-1.0 \mathrm{nmol}$ of total carnitine), and individual acylcarnitines were detected and quantitated by reverse-phase HPLC coupled to an instream continuous flow through a beta counter (FloOne, Radiomatic, Canberra-Packard, Tampa, FL). A pretreatment of the samples by means of solid-phase extraction was used to avoid the underestimation of the esterified fractions due to the presence of high concentrations of free carnitine (14). Samples were applied to Sep-Pak $\mathrm{C}_{18}$ cartridges (Waters, Millipore Corp., Milford, MA) equilibrated with $5 \mathrm{mM}$ acetic acid. Free carnitine, acetylcarnitine, and propionylcarnitine were eluted with $8 \mathrm{~mL}$ of $5 \mathrm{mM}$ acetic acid. Longer chain (>C4) acylcarnitines were eluted by methanol. The recovery of carnitine esters in the eluate was complete. Isovalerylglycine and 3-hydroxyisovaleric acid were measured by gas chromatography-mass spectrometry by following common procedures of extraction and derivatization (15).

Calpain and calpastatin activities in erythrocytes. Blood samples, immediately after collection from an antecubital vein, were washed four times with an isotonic solution (pH 7.4) containing $10 \mathrm{mM} \mathrm{N}$-2-hydroxyethylpiperazine$N^{\prime}$-2-ethanesulfonic acid, $0.14 \mathrm{M} \mathrm{NaCl}, 5 \mathrm{mM} \mathrm{KCl}$, and 5 $\mathrm{mM}$ glucose. Leukocytes and platelets were removed by using a modification (10) of the filtration procedure reported by Beutler et al. (16). Packed erythrocytes $(2 \mathrm{~mL})$

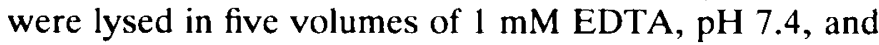
centrifuged at $24000 \times \mathrm{g}$ for $10 \mathrm{~min}$. The membrane fraction was discarded, and the clear supernatant (cytosolic fraction) was collected and dialyzed against $50 \mathrm{mM}$ $\mathrm{Na}$ acetate buffer, at $\mathrm{pH} 6.4$, containing $1 \mathrm{mM}$ EDTA and $0.5 \mathrm{mM} 2$-mercaptoethanol. A sample containing $200 \mathrm{mg}$ of $\mathrm{Hb}$ was submitted to diethylaminoethyl ion exchange chromatography on a column $(1 \times 10 \mathrm{~cm})$ of diethyaminoethyl-cellulose 50 equilibrated in the above dialysis medium, and the absorbed proteins were eluted by a linear gradient of $\mathrm{NaCl}$ (from 0 to $0.3 \mathrm{M}$ ). Aliquots (50 $\mathrm{mL}$ ) of the eluted fractions were heated at $90^{\circ} \mathrm{C}$ for $3 \mathrm{~min}$, then assayed for calpain and calpastatin. These substances were eluted in separate peaks $(17,18)$, and from the areas of these peaks their individual concentrations were computed.

Calpain activity was assayed as previously described (19). One unit is defined as the amount of enzyme releasing $1 \mathrm{nmol}$ of free amino groups per hour under the assay conditions.

Calpastatin inhibition was measured as previously reported (19). One unit of calpastatin activity is defined as the amount that inhibits one unit of calpain activity.

Phosphorylation of erythrocyte inside-out vesicle proteins. Erythrocyte inside-out vesicles $(0.1 \mathrm{mg}$ protein), prepared as previously described (20), were incubated at

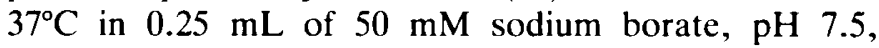
containing $5 \mu \mathrm{M} \mathrm{MgCl}_{2}$ and $10 \mu \mathrm{M}$ labeled ATP $(1 \mathrm{Ci} /$ $\mathrm{mmol})$. At the times indicated, aliquots $(0.05 \mathrm{~mL})$ were withdrawn and the reaction was stopped by addition of $0.05 \mathrm{~mL}$ of $20 \mathrm{mM}$ Tris- $\mathrm{HCl}, \mathrm{pH} 8.0$, containing $2 \%$ SDS, $40 \%$ glycerol, $2 \mathrm{mM}$ EDTA, and $80 \mathrm{mM}$ 2-mercaptoethanol, followed by heating at $90^{\circ} \mathrm{C}$ for $3 \mathrm{~min}$. Samples were then submitted to polyacrylamide slab gel electrophoresis (21). The gels were stained with Coomassie brilliant blue, destained with $7 \%$ acetic acid containing $10 \%$ ethanol, and dried. The labeled proteins were identified by autoradiography of dried gels.

Loading of human erythrocyte with $\mathrm{Ca}^{2+}$ and measurement of erythrocyte fragility. To erythrocytes $(3 \mathrm{~mL}$ of packed cells), prepared as described above, were added 3 $\mathrm{mL}$ of $10 \mathrm{mM} N$-2-hydroxyethylpiperazine- $N^{\prime}$-2-ethane- 
sulfonic acid, $\mathrm{pH} 7.4$, containing $5 \mathrm{mM} \mathrm{KCl}, 140 \mathrm{mM}$ $\mathrm{NaCl}$, and $5 \mathrm{mM}$ glucose. Aliquots $(1 \mathrm{~mL})$ were incubated at $37^{\circ} \mathrm{C}$ with $5 \mu \mathrm{M} \mathrm{A23187}$ in the absence of $\mathrm{Ca}^{2+}$ or in the presence of increasing concentrations of $\mathrm{Ca}^{2+}$. The cells were incubated for $30 \mathrm{~min}$ and then collected by centrifugation at $800 \times g$ for $10 \mathrm{~min}$. The $\mathrm{Hb}$ content in the clear supernatant was determined by the absorbance at $540 \mathrm{~nm}$ (22).

\section{RESULTS}

The amount of the carnitine fraction in blood plasma and in urine of the patient at the end of carnitine and glycine treatments is reported in Table 1. Despite the large amount of carnitine given $(200 \mathrm{mg} / \mathrm{kg} / \mathrm{d})$, the free carnitine concentration in the plasma $(13 \mu \mathrm{mol} / \mathrm{L})$ was lower than that found in untreated normal subjects (31 $\mu \mathrm{mol} / \mathrm{L}$ ), although the esterified carnitine concentration was significantly higher (21 versus $7 \mu \mathrm{mol} / \mathrm{L})$.

These results are in agreement with those previously obtained in other cases of isovaleric acidemia $(8,9)$. We have, in addition, established that the dominant carnitine ester in blood and urine after carnitine treatment is indeed IVC. This was still true after glycine treatment, although the levels were lower, especially in the urine. In contrast, IVC was undetectable in the control. Because IVC readily diffuses across cell membranes, erythrocyte calpain must be exposed to the same IVC concentration as plasma.

The ratio of free to esterified carnitine in plasma was also much lower after carnitine or glycine treatment than in the controls. This is partly because, after $6 \mathrm{mo}$ of glycine treatment, the free carnitine concentration in plasma was well below that of controls, perhaps because endogenous carnitine is used in preference to glycine for removing excess isovaleryl groups. This would be concordant with the different published values for the affinity of isovalerylCoA toward carnitine acetyl transferase $(\mathrm{Km}$ $<0.05 \mathrm{mM})(23)$ and toward glycine- $\mathrm{N}$-acylase $(\mathrm{Km}>$ $0.5 \mathrm{mM})(24)$.

The elimination of carnitine with urine (Table 1) at the end of the carnitine supplementation period shows that IVC represents the most abundant fraction, accounting for $85.1 \%$ of total carnitine and $97.9 \%$ of esterified carnitine. After the glycine supplementation period, urinary excretion of carnitine was greatly reduced even in comparison with that of normal subjects. However, even in

Table 1. Concentrations of carnitine and its esters in blood plasma and urine after giving the patient carnitine or glycine

\begin{tabular}{lccc}
\hline & Free & Isovalerylester & Other esters \\
\hline Plasma ( $\mu$ mol/L) & & & \\
After carnitine & 13 & 19 & 2 \\
After glycine & 7 & 5 & 1 \\
Control & 31 & $\mathrm{ND}^{*}$ & 7 \\
Urine $(\mu \mathrm{mol} / \mathrm{g}$ creatinine) & & & \\
After carnitine & 382 & 2500 & 55 \\
After glycine & 18.2 & 45.4 & 9.0 \\
\hline
\end{tabular}

* ND, not determined. this condition, IVC represented the most abundant fraction. These data indicate the severe carnitine deficiency in the patient in the absence of carnitine treatment and show that administered carnitine was almost entirely used to drain the intramitochondrial accumulated isovaleryls.

Table 2 shows the activity of calpain of the red blood cells collected from the patient after 6 mo of a daily supplementation of glycine. The activity of the enzyme was significantly lower than that in erythrocytes of a normal subject of the same age (control) as well as lower than that in the erythrocytes of the same patient 6 mo after ending carnitine supplementation and replacing it with glycine supplementation.

Carnitine treatment did not greatly affect the activity of calpastatin, which was similar to that found in the control.

After L-carnitine treatment, the rates of erythrocyte band-3 phosphorylation were about twice those obtained after glycine treatment or in the control (Fig. 1). This increased phosphorylation, which is concomitant with calpain autodigestion, is attributed to the unmasking of new phosphorylation sites $(6,25)$. Our results are therefore additional evidence for activation of calpain by IVC in vivo.

An increase in erythrocyte band-3 phosphorylation increases the lytic effect of $\mathrm{Ca}^{2+}$ in the presence of the $\mathrm{Ca}$ ionophore A-23187 (6) (Fig. 2). Indeed, after carnitine treatment, the cells lysed with increasing $\mathrm{Ca}^{2+}$ concentration much more readily than in the control or after glycine treatment.

\section{DISCUSSION}

The metabolic defect producing isovaleric acidemia (26) is a defiency of isovaleryl-CoA dehydrogenase (27). This results in the accumulation of isovaleryl-CoA in mitochondria with a consequent deficiency of free CoA.

Transfer of the excess isovaleryl groups by conjugation with either glycine $(28,29)$ or carnitine $(8,9,25)$ has been proposed as a way to restore the CoA level. The isovalerylglycine and IVC formed, unlike isovaleryl-CoA itself, readily diffuse across mitochondrial and cellular membranes to be excreted in the urine. Without treatment or after treatment with glycine, endogenous carnitine is rapidly depleted by this conjugation (30), giving a low level of free carnitine itself and increased acyl-carnitine/ free carnitine ratios in plasma and urine. Carnitine synthesis is evidently inadequate to maintain a level of free

Table 2. Calpain and calpastatin levels in erythrocytes*

\begin{tabular}{lcc}
\hline & $\begin{array}{c}\text { Calpain } \\
(\mathrm{U} / \mathrm{mg} \mathrm{Hb})\end{array}$ & $\begin{array}{c}\text { Calpastatin } \\
(\mathrm{U} / \mathrm{mg} \mathrm{Hb})\end{array}$ \\
\hline Control & $170 \pm 23$ & $303 \pm 25$ \\
Carnitine-treated patient & 96 & 260 \\
Glycine-treated patient & 140 & 255 \\
\hline
\end{tabular}

* The values of control subjects (mean \pm SD) were obtained by assays on five different normal subjects. The values for treated patients are the mean of four different determinations. 


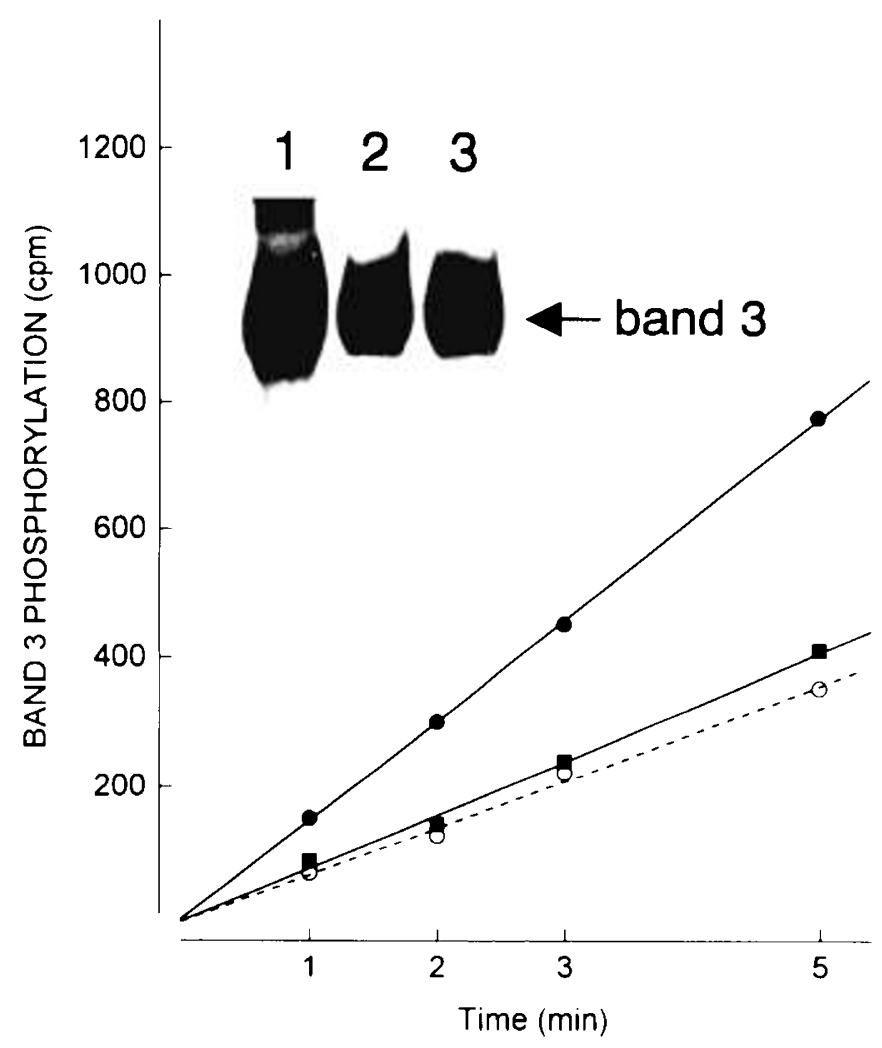

Figure 1. ${ }^{32} \mathrm{P}$ incorporation in the band-3 region of erythrocyte insideout vesicles from control subjects $(0 \ldots-0$ : lane $l$ in the insert) or the patient after treatment with carnitine (-) lane 2) or glycine $(\mathbf{Q}-\mathbf{a}$; lane 3). Erythrocyte inside-out vesicles were incubated at $37^{\circ} \mathrm{C}$ in the presence of $10 \mu \mathrm{M}$ labeled ATP $(1 \mathrm{Ci} / \mathrm{mmol})$. At the times indicated, the reaction was stopped as described in Methods. After heating at $90{ }^{\circ} \mathrm{C}$ for 3 min, samples were submitted to polyacrylamide slab gel electrophoresis. The labeled proteins were identified by autoradiography of dried gels as shown in the insert. The relative bands were then cut and counted by liquid scintillation, allowing the quantitation of ${ }^{32} \mathrm{P}$ incorporation, which is shown in the lower panel.

carnitine sufficient for the conjugation of the excess isovaleryls, and this also accounts for the fact that loading with carnitine induces a remarkable increase both in the blood level of IVC and in its urinary excretion.

The continuous exposure of erythrocytes to elevated IVC levels can activate their calpain (6), principally by inducing a 10 -fold increase in affinity toward $\mathrm{Ca}^{2+}$, the binding of which is necessary for its activity. This activation also initiates calpain autodigestion and eventually leads to destruction of the enzyme. The relative stability of erythrocyte calpastatin, the natural inhibitor of calpain, further supports the previous results showing that in vitro IVC activates calpain despite the presence of calpastatin (6). In other terms, IVC releases calpain activity from the calpastatin control. This validates the suggestion of an initial activation by IVC on the "calpaincalpastatin'" system.

This scenario plausibly accounts for our observation that there was a decrease of erythrocyte calpain activity in our patient after carnitine but not after glycine treatment. The decrease is also consistent with the increased rate of erythrocyte band -3 phosphorylation, found only

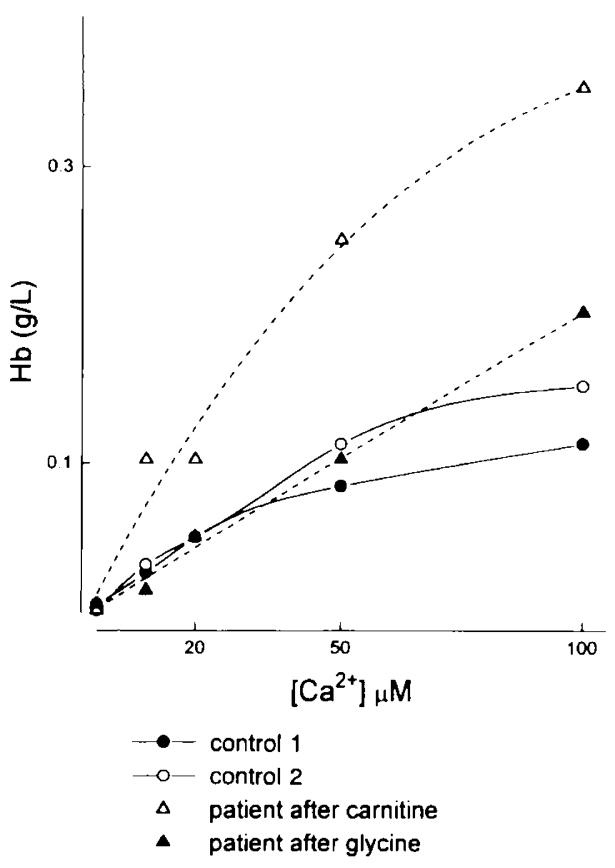

Figure 2. Effect of $\mathrm{Ca}^{2+}$ loading on the lysis of erythrocytes from control subjects and from the patient after treatment with carnitine or glycine. Erythrocytes were incubated with $5 \mu \mathrm{M}$ ionophore $\mathrm{A}-23187$ in the presence of the indicated concentrations of $\mathrm{Ca}^{2+}$, as described in Methods.

after carnitine treatment, which we believe is probably caused by unmasking of new phosphorylation sites. Their appearance may be a prelude to the degradation of certain membrane glycoproteins $(22,25)$, thus accounting for the higher susceptibility of erythrocytes after carnitine treatment to lysis induced by calcium loading in the presence of a calcium ionophore.

All these effects are therefore seen as secondary to the initial calpain activation by IVC and may be significant from both a diagnostic and a prognostic point of view. The decline in calpain activity does not therefore seem to be injurious, and indeed it could be part of a complex and as yet unknown mechanism linking carnitine with metabolic improvement. Nevertheless, measurement of erythrocyte fragility can be appropriate for the follow-up of patients receiving carnitine treatment.

Acknowledgment. The authors thank Prof. Peter Jocelyn for the critical reading of the manuscript.

\section{REFERENCES}

1. Pontremoli S, Melloni E 1986 Fxtralysosomal protein degradation. Ann Rev Biochem 55:455-581

2. Suzuki K. Imajoh S, Emori Y, Kawasaki H, Minami Y, Ohno S 1987 Calcium-activated neutral protease and its endogenous inhibitor. Activation at the cell membrane and biological function. FEBS Let1 220:271-277

3. Mellgren RL 1987 Calcium-dependent proteases: an enzyme system active at cellular membranes? FASEB J 1:110-115

4. Murachi T 1989 Intracellular regulating system involving calpain and calp. astatin. Biochem Int 18:26,3-294

5. Johnson P 1990) (alpain (intracellular calcium-activated cysteine proteinases): structure-activity relationships and involvement in normal and abnormal cellular metabolism. Int J Biochem 22:811-822

6. Pontremoli S, Melloni E. Michetti M, Sparatore B. Salamino F, Siliprandi N, Horecker BL. 1987 Isovalerylearnitine is a specific activator of calpains of human neutrophils. Biochem Biophys Res Commun 148:1189-1195 
7. Pontremoli S, Melloni E, Viotti PL, Michetti M, Di Lisa F, Siliprandi N 1990 Isovalerylcarnitine is a specific activator of the high calcium requiring calpain form. Biochem Biophys Res Commun 167:373-380

8. Roe CR, Millington DS, Maltby DA, Kahler SG, Bohan TP 1984 L-Carnitine therapy in isovaleric acidemia. J Clin Invest 74:2290-2295

9. de Sousa C, Chalmers RA, Stacey TE, Tracey BM, Weaver CM, Bradley D 1986 The response to L-carnitine and glycine therapy in isovaleric acidaemia. Eur J Pediatr 144:451-456

10. Melloni E, Salamino F, Sparatore B, Michetti M, Pontremoli S $1984 \mathrm{Ca}^{2+}$ dependent neutral proteinase from human erythrocytes: activation by $\mathrm{Ca}^{2+}$ ions and substrate and regulation by the endogenous inhibitor. Biochem Int 8:477-489

11. Parvin R, Pande SV 1977 Microdetermination of $(-)$ carnitine and carnitine acetyltransferase activity. Anal Biochem 79:190-201

12. Pande SV, Caramancion N 1981 A simple radioisotopic assay of acetylcarnitine and acetyl-CoA at picomolar levels. Anal Biochem 112:30-38

13. Kerner J, Bieber LL 1985 Isolation and identification of methyloctanoylcarnitines from human urine. Prep Biochem 15:237-257

14. Di Lisa F, Bieber LL, Kerner J, Menabö R, Barbato R, Siliprandi N 1992 Methods for carnitine assay. In: Ferrari R, Di Mauro S, Sherwood G (eds) L-Carnitine and Its Role in Medicine: From Function to Therapy. Academic Press, London, pp 37-61

15. Dionisi-Vici C, Burlina AB, Bertini E, Bachmann C, Mazziotta M, Zacchello F, Sabetta G, Hale D 1991 Progressive neuropathy and recurrent myoglobinuria in a child with long chain 3-hydroxyacyl-CoA dehydrogenase deficiency. J Pediatr 118:744-746

16. Beutler E, West C, Blume KG 1976 The removal of leukocytes and platelets from whole blood. J Lab Clin Med 88:328-333

17. Pontremoli S, Melloni E, Sparatore B, Salamino F, Viotti PL, Michetti M. Duzzi L. Bianchi G 1986 Decreased level of calpain inhibitor activity in red blood cells from Milan hypertensive rats. Biochem Biophys Res Commun 138:1370-1375

18. Pontremoli S, Melloni E, Sparatore B, Salamino F, Michetti M, Pontremoli R. Tizianello A, Barlassina C, Cusi D, Colombo R, Bianchi G 1988 Erythrocyte deficiency in calpain inhibitor activity in essential hypertension. Hypertension 12:474-478

19. Melloni E, Sparatore B, Salamino F, Michetti M, Pontremoli S 1982 Cytosolic calcium dependent proteinase of human erythrocytes: formation of an enzyme-natural inhibitor complex induced by $\mathrm{Ca}^{2+}$ ions. Biochim Biophys Res Commun 106:731-740

20. Pontremoli S, Melloni E, Sparatore B, Michetti M, Horecker BL 1984 A dual role for the $\mathrm{Ca}^{2+}$-requiring proteinase in the degradation of hemoglobin by erythrocyte membrane proteinases. Proc Natl Acad Sci USA 81:6714-6717

21. Fairbanks G, Steck TL, Wallach DFH 1971 Electrophoretic analysis of the major polypeptides of the human erythrocyte membrane. Biochemistry 10:2606-2617

22. Pontremolis, Melloni E, Sparatore B, Salamino F, Pontremoli R, Tizianello A 1987 Increased phosphorylation in red cell membranes of subjects affected by essential hypertension. Biochem Biophys Res Commun 145:1329-1334

23. Chase JFA 1967 The substrate of carnitine acetyltransferase. Biochem J $104: 510-518$

24. Kolvraa S, Gregersen N 1986 Acyl-CoA:glycine N-acyltransferase: organelle localization and affinity toward straight- and branched-chained acyl-CoA esters in rat liver. Biochem Med 36:98-105

25. Melloni E, Pontremoli S 1989 The calpains. Trends Neurol Sci 12:438-444

26. Tanaka K, Isselbacher KJ 1967 The isolation and identification of $\mathrm{N}$-isovalerylglycine from urine of patients with isovaleric acidemia. J Biol Chem 242:2966-2972

27. Rhead WJ, Tanaka K 1980 Demonstration of a specific mitochondrial isovaleryl-CoA dehydrogenase deficiency in fibroblasts from patients with isovaleric acidemia. Proc Natl Acad Sci USA 77:580-583

28. Krieger I, Tanaka K 1976 Therapeutic effect of glycine in isovaleric acidemia. Pediatr Res 10:25-29

29. Naglak M, Salvo R, Madsen K, Dembure P, Elsas L, 1988 The treatment of isovaleric acidemia with glycine supplement. Pediatr Res 24:9-13

30. Chalmers RA, Roe CR, Stacey TE, Hoppel CL 1984 Urinary excretion of L-carnitine and acylcarnitines by patients with disorders of organic acid metabolism: evidence for secondary insufficiency of L-carnitine. Pediatr Res 18:1325-1328 